\title{
Relationship between sperm morphology and sperm DNA dispersion
}

\author{
Joanna Jakubik-Uljasz ${ }^{1}$, Kamil Gill ${ }^{1}$, Aleksandra Rosiak-Gill ${ }^{1,2}$, Malgorzata Piasecka $^{1}$ \\ ${ }^{1}$ Department of Histology and Developmental Biology, Pomeranian Medical University in Szczecin, Szczecin, Poland; ${ }^{2}$ VitroLive Fertility Clinic in \\ Szczecin, Szczecin, Poland \\ Contributions: (I) Conception and design: J Jakubik-Uljasz, K Gill, M Piasecka; (II) Administrative support: None; (III) Provision of study materials or \\ patients: None; (IV) Collection and assembly of data: J Jakubik-Uljasz, K Gill, A Rosiak-Gill; (V) Data analysis and interpretation: J Jakubik-Uljasz, \\ M Piasecka; (VI) Manuscript writing: All authors; (VII) Final approval of manuscript: All authors. \\ Correspondence to: Prof. Małgorzata Piasecka. Department of Histology and Developmental Biology, Pomeranian Medical University in Szczecin, str. \\ Żołnierska 48, zip code 71-210, Poland. Email: mpiasecka@ipartner.com.pl.
}

Background: The pathogenesis of teratozoospermia ( $<4 \%$ morphologically normal sperm cells) and the relationship between sperm morphological abnormalities and abnormal sperm nuclear DNA fragmentation, which are considered indicators of male fertility, have not been elucidated. Our research was designed to determine the prevalence of different sperm DNA fragmentation (SDF) levels in men with teratozoospermia and to establish a discriminating threshold value for SDF in assessing sperm morphology.

Methods: Basic semen characteristics and detailed sperm morphological analysis (head, neck, midpiece, and tail defects and excess residual cytoplasm) (WHO, 2010), and the nuclear sperm DNA dispersion test were performed on semen samples obtained from 523 men with teratozoospermia $(n=296)$ and those without teratozoospermia ( $\mathrm{n}=227)$.

Results: Subjects with abnormal sperm morphology had not only lower results for standard sperm characteristics, including detailed sperm morphological abnormalities, but also a higher proportion of sperm cells with SDF vs. men with normal sperm morphology. Moreover, significantly fewer subjects with low SDF levels $(\leq 15 \%)$, more subjects with high SDF levels (>30\%) and a higher odds ratio (OR) for having high SDF levels were found in the group of men with teratozoospermia $v s$. men without teratozoospermia. However, the receiving operating characteristic (ROC) curve analysis indicated that a SDF $>18 \%$ was a significant negative predictive value to distinguish between men with normal sperm morphology or men with abnormal sperm morphology. The optimal area under the ROC curve (AUC) was 0.746. In the group of men with teratozoospermia, a higher incidence of men with $>18 \%$ SDF and a higher OR for having $>18 \%$ SDF were observed. SDF negatively correlated with sperm number, morphologically normal sperm cells, sperm motility and sperm vitality but positively correlated with the teratozoospermia index (TZI) and detailed sperm morphological abnormalities.

Conclusions: The obtained findings demonstrated that: (I) detailed sperm structural defects coexist with abnormal nuclear sperm DNA dispersion, (II) men with teratozoospermia may have a higher risk for sperm DNA damage, (III) the calculated optimal SDF value of $18 \%$ measured by the DNA sperm dispersion test is the best criterion to predict normal and abnormal sperm morphology.

Keywords: Male infertility; semen analysis; sperm morphology; sperm DNA fragmentation (SDF)

Submitted Oct 03, 2019. Accepted for publication Jan 06, 2020.

doi: $10.21037 /$ tau.2020.01.31

View this article at: http://dx.doi.org/10.21037/tau.2020.01.31 


\section{Introduction}

Infertility has become a global health problem, with approximately $15-20 \%$ of couples worldwide having problems achieving pregnancy. Male factors contribute to approximately $20-70 \%$ of infertility cases, and almost $30 \%$ of these factors have a direct impact on fertility (1-3). The first step in assessing male infertility is conventional semen analysis according to World Health Organization standards (4). It is known that this semen analysis has a relatively limited diagnostic value in the assessment of sperm fertilization competency due to multiple types of sperm defects, including molecular abnormalities, which cannot be verified by conventional analysis. Therefore, there is a need to introduce new diagnostic tools to expand the standard semen assessment (2,5-8).

Sperm morphological abnormalities and abnormal sperm chromatin maturity can be considered as indicators of male fertility (9-16). Therefore, the assessment of sperm structural abnormalities and sperm DNA damage is very important during the evaluation of male fertility potential. However, the pathogenesis of these disorders and the relationships between them are not fully understood because sperm morphogenesis, including high compaction of spermatids chromatin, is unique and complex $(17,18)$. These processes lead to the creation of specific sperm structures, which are required for oocyte fertilization. Morphogenesis failure can provoke not only sperm structural defects and diminished sperm maturity but also sperm nuclear DNA fragmentation (SDF) due to impaired repair of nuclear DNA strand breaks during the early spermatid stage. Furthermore, sperm with diminished maturity are known to generate higher levels of reactive oxygen species and have increased DNA fragmentation. Finally, oxidative stress, 'abortive' apoptosis of differentiated germinal cells and teratozoospermia can appear (19-23). It should be highlighted that ejaculated sperm cells with head defects often have abnormal condensed chromatin and denatured nuclear DNA $(9,12,14-16)$. It is difficult to determine whether morphological disorders directly contribute to sperm nuclear DNA strand breaks or whether failure in chromatin remodelling promotes the formation of abnormal sperm head defects; it can only be assumed that these factors coexist (9-11,13-16,19) (Figure 1). Therefore, our research was designed to find the relationship between sperm morphology and sperm DNA dispersion, to determine the prevalence of different SDF levels in men with teratozoospermia and to establish a discriminating threshold value for SDF in assessing sperm morphology.

\section{Methods}

\section{Study population}

The study group comprised 523 men (median age: 32.00 years) who visited the Department of Histology and Developmental Biology (Pomeranian Medical University in Szczecin, Poland) to evaluate their semen parameters. Men were divided into two groups: 227 men with normal sperm cell morphology $\geq 4 \%$ (median age: 30.00 years) as the control group and 296 men with normal sperm cell morphology $<4 \%$ (teratozoospermic subjects) (median age: 33.00 years) as the experimental group. These cohort groups originated from general population of men. The men with: a history of mumps, co-existing systemic disease; varicocele, testicular injury or cancer, cryptorchidism, a history of testicular torsion and a clinical picture suggestive of obstructive azoospermia were excluded from the study.

Normozoospermia $(\mathrm{n}=205)$ was considered according to the following criteria (WHO, 2010): sperm progressive motility $\geq 32 \%$, sperm concentration $\geq 15 \mathrm{mln} / \mathrm{mL}$, total sperm count $\geq 39 \mathrm{mln}$, and sperm morphology $\geq 4 \%$. In the study group, the following abnormal seminological categories were distinguished: oligozoospermia (men with an abnormal number of sperm cells, $n=16$ ); asthenozoospermia (men with abnormal sperm motility, $\mathrm{n}=5$ ); teratozoospermia (men with abnormal sperm morphology, $\mathrm{n}=115$ ); oligoasthenozoospermia (men with an abnormal number and motility of sperm cells, $n=1)$; oligoteratozoospermia (men with an abnormal number and morphology of sperm cells, $n=64$ ); asthenoteratozoospermia (men with abnormal sperm motility and morphology, $n=40$ ); and oligoasthenoteratozoospermia (men with an abnormal number, motility and morphology of sperm cells, $\mathrm{n}=77$ ).

\section{Standard semen analysis}

Standard semen analysis, including macroscopic and microscopic assessment of the semen was performed as recommended by the World Health Organization (WHO) guidelines (4). Subjects were asked to abstain from ejaculating for 2-7 days prior to sample collection. The specific length of the abstinence time was recorded. All semen samples were incubated at $37^{\circ} \mathrm{C}$ immediately upon donation and were liquefied for $30 \mathrm{~min}$ before analysis. The sperm concentration (with an improved Neubauer 


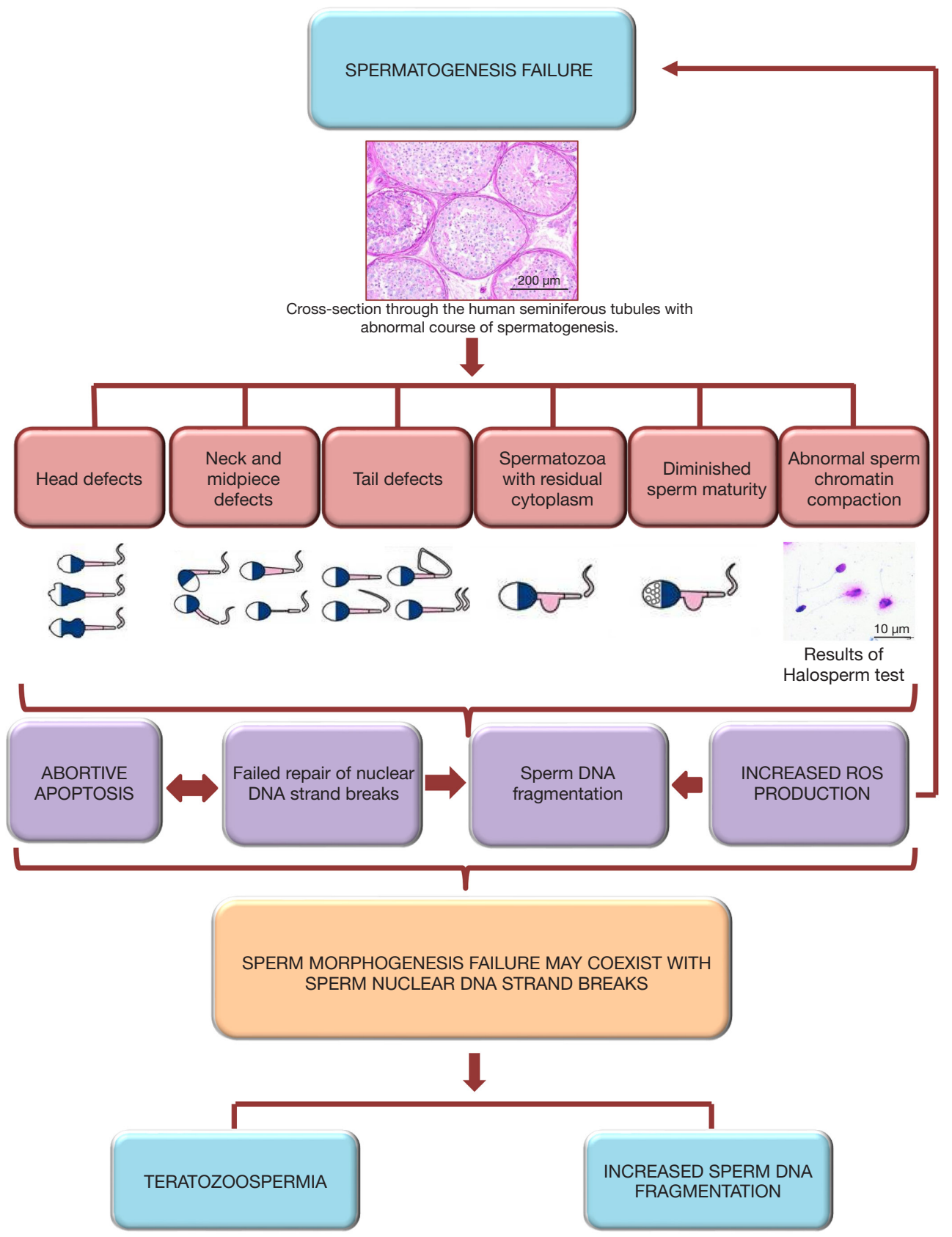

Figure 1 Hypothesized mechanism leading to the simultaneous creation of sperm morphological defects and sperm chromatin abnormalities. Abnormal spermatogenesis can result in (I) an increased number of sperm morphological defects, often leading to teratozoospermia; (II) diminished sperm maturity; (III) impaired sperm chromatin compaction; (IV) failed repair of nuclear DNA strand breaks appearing during the early spermatid stage; and (V) failure of germinal cells to undergo apoptosis, namely, 'abortive' apoptosis, meaning that germinal cells escape apoptosis and may display nuclear DNA fragmentation. Nonmature spermatozoa or sperm cells with diminished maturity (e.g., spermatozoon with residual cytoplasm) are known to have a higher number of resistant nuclear DNA strand breaks and to produce higher levels of reactive oxygen species, which can secondarily cause sperm DNA fragmentation and, finally, spermatogenesis failure (19-23). 
Table 1 Criteria of sperm detailed morphological analysis according to WHO 2010 and based on developed morphogram (24)

\begin{abstract}
Morphologically normal spermatozoa
Sperm head: length $4.10 \mu \mathrm{m}$, width $2.80 \mu \mathrm{m}$, length-to-width ratio $1.50 \mu \mathrm{m}$; acrosomal region comprising $40-70 \%$ of normal head area; pale vacuoles ( $\leq 2)$ occupying $\leq 20 \%$ of the normal head area, the post-acrosomal region should not contain any vacuoles

Sperm midpiece: the same length as the sperm head; length $4.00 \mu \mathrm{m}$, width $0.60 \mu \mathrm{m}$

Sperm tail: length $45.00 \mu \mathrm{m}$

Morphologically abnormal spermatozoa

Head defects: large or small oval shape; large or small non-oval shape; tapered; pyriform; amorphous; vacuolized: in the acrosomal region $>2$ vacuoles occupying $>20 \%$ of the head area; post-acrosomal region should not contain any vacuoles; small acrosome area: $<40 \%$ of the head area; big acrosome: $>70 \%$ of the head area; no acrosome
\end{abstract}

Abnormal head-tail attachment/junction: pinhead sperm; decapitated sperm (detached tail, acephalic sperm)

Neck and midpiece defects: bent neck; asymmetrical; thick insertion; thin

Tail defects: short; bent; coiled; double/multiply

Spermatozoa with excess residual cytoplasm: $\geq 1 / 3$ of the sperm head size, absence

haemocytometer, Heinz Hernez Medizinalbedarf GmbH, Hamburg, Germany), motility (total and progressive motility), vitality [live sperm cells: eosin-negative or hypo-osmotic-reactive sperm cells (HOS) test] and the concentration of leukocytes (peroxidase-positive cells, the Endtz test, LeucoScreen kit, FertiPro N.V., Beernem, Belgium) were assessed with phase-contrast microscope (Primo Star, Zeiss, Germany), whereas the sperm morphology and teratozoospermia index (TZI) (number of spermatozoa with $\geq 1$ head defect, midpiece defect, or principal piece defect and the number of sperm cells with excess residual cytoplasm/number of abnormal spermatozoa) were evaluated with a bright light microscope (CX 31 Olympus Optical Co., Ltd., Tokyo, Japan). The TZI reflects the multiple defects per abnormal spermatozoon. TZI is in the range of 1 to 4 . This means, that one spermatozoon can contain a single defect, or in a combination of two, three or all four defects simultaneously.

\section{Detailed sperm morphological assessment}

Sperm morphology was analysed on the basis of the Papanicolaou-stained smears (WHO, 2010), while structural abnormalities of male reproductive cells and the TZI were evaluated according to the developed morphogram (24). Criteria of sperm detailed morphological analysis, according to WHO 2010, are described in Table 1.

\section{Sperm chromatin dispersion (SCD) test (Halosperm $^{\circledast}$ test)}

After liquefaction, an aliquot of $100 \mu \mathrm{L}$ of the raw semen sample was used for the SCD test. The method used was the Halosperm ${ }^{\circledR}$ G2 kit (Halotech DNA, Madrid, Spain) in keeping with the manufacturer's protocol: immersion of sperm cells in an inert agarose microgel on a pretreated slide, acid denaturation of DNA, removal of most of the nuclear proteins by lysis, dehydration in $70 \%$ and $100 \%$ alcohol, and staining with eosin and thiazine. Next, a minimum of 300 spermatozoa per sample were scored under the $100 \times$ objective of the bright light microscope (CX 31 Olympus Optical Co., Ltd., Tokyo, Japan).

The Halosperm test is based on principle that sperm with nonfragmented DNA produce a large halo of dispersed loops. A large halo is defined as a halo width that is similar to or longer than the diameter of the sperm head. A medium-sized halo is defined as a halo width that is $>1 / 3$ the diameter of the sperm head. Spermatozoa with fragmented DNA produce a small-sized halo, defined as a halo width that is $\leq 1 / 3$ the diameter of the sperm head, or no halo. Sperm cells can also be degraded and have irregularly, weakly stained heads. The results are presented as the percentage of spermatozoa with a small or nonexistent halo, namely, a degraded halo, in the total number of assessed sperm cells $(25,26)$. 


\section{Statistical analysis}

The statistical analyses were performed using the software Statistica version 13.0 (StatSoft, Poland) and MedCalc version 15.2.2 (MedCalc Software, Belgium). The conformity of variables with the normal distribution was examined using the Shapiro-Wilk test assuming compatibility at $\mathrm{P}>0.05(>5 \%)$. To compare two independent groups, the nonparametric Mann-Whitney $\mathrm{U}$ test was utilized. A Chi-square test was used to analyse the categorical data. The interdependences of variables were examined by calculating Spearman's rank-order correlation coefficient $\left(\mathrm{r}_{\mathrm{s}}\right)$. To interpret the strength association between the study parameters, the following levels of correlation were presumed: $<0.2$ lack of linear dependence, $0.2-0.4$ weak dependence, 0.4-0.7 moderate dependence, 0.7-0.9 strong dependence, and $>0.9$ very strong dependence. The predictive values of the analysed variables were verified using the receiver operating characteristic (ROC) curve and the area under the curve (AUC), taking into account the standard error (SE) and $95 \%$ confidence interval (95\% CI). The AUCs were as follows: 0.9-1.0 excellent predictive value, $>0.8-0.9$ good predictive value, $>0.7-0.8$ satisfactory predictive value, $>0.6-0.7$ moderate predictive value, and $0.5-0.6$ insufficient predictive value. The findings were considered statistically significant at $\mathrm{P}<0.05$.

\section{Results}

\section{Standard semen characteristics and detailed sperm morphological analysis}

Statistically significant differences were observed between men with abnormal sperm morphology and men with normal sperm morphology in terms of the semen volume $(\mathrm{P}=0.0002)$, sperm concentration, total sperm count, sperm progressive, nonprogressive motility and total motility, as well as vitality $(\mathrm{P}<0.00001)$. The study groups also differed significantly in terms of the TZI; the percentages of morphologically normal sperm cells, those with sperm cell head defects, those with neck and midpiece defects, and those with tail defects; and the percentage of spermatozoa with excess residual cytoplasm $(\mathrm{P}<0.00001)$ (Mann-Whitney U-test) (Table 2).

\section{Analysis of sperm nuclear DNA dispersion}

According to data obtained by some authors (2,27-35), the levels of sperm chromatin damage were estimated based on the following criteria: $0-15 \%$ SDF (low levels of sperm cells with fragmented DNA, high fertility potential), 16-30\% SDF (moderate levels, moderate fertility potential) and $>30 \%$ SDF (high levels, low fertility potential).

The percentage of SDF was significantly higher in men with teratozoospermia $v s$. men with normal sperm morphology (median: $22.00 \%$ vs. $13.00 \%)(\mathrm{P}<0.00001$, Mann-Whitney U-test) (Table 2). Moreover, a lower proportion of men $(22.97 \%)$ with low SDF levels and a higher incidence of men $(32.77 \%)$ with high SDF levels were found among men with teratozoospermia $v s$. men with normal sperm cell morphology $(58.60 \%, 4.84 \%$, respectively) $(\mathrm{P}<0.0001)$ (Table 3). Men with abnormal sperm cell morphology also had a significantly lower odds ratio (OR) for having low SDF levels (OR: 0.2175) and a higher OR for having high SDF levels (OR: 9.5715) $(\mathrm{P}<0.0001)\left(\mathrm{Chi}^{2}\right.$ test) (Table 4).

The ROC analysis revealed the significant and satisfactory negative predictive value of SDF (18\%) for sperm morphology. The optimal area under the ROC curve was $0.746(\mathrm{P}<0.001$, sensitivity $62.84,100$-specificity 73.13) (Figure 2). Based on the ROC results, a lower proportion of men $(37.16 \%)$ with $\leq 18 \%$ SDF and a higher incidence of men $(62.84 \%)$ with $>18 \%$ SDF were found among men with teratozoospermia $v s$. men with normal sperm cell morphology (73.12\%, 26.88\%, respectively) $(\mathrm{P}<0.0001)$ (Table 5). Furthermore, men with teratozoospermia had a significantly lower OR for having SDF levels $<18 \%$ (OR: 0.2173 ) and a higher OR for showing SDF levels $>18 \%$ (OR: 4.6015) $(\mathrm{P}<0.0001)\left(\mathrm{Chi}^{2}\right.$ test $)($ Table 6).

\section{Correlation between sperm nuclear DNA dispersion and semen characteristics}

SDF negatively correlated with sperm concentration, total sperm count, sperm morphology, progressive sperm motility, total sperm motility and sperm vitality $(\mathrm{P}=0.00001)$ but positively correlated with the TZI; the percentages of immotile sperm cells, head defects, midpiece defects and 
Table 2 Descriptive statistics and comparisons of standard semen parameters and SDF levels between study groups

\begin{tabular}{|c|c|c|}
\hline Parameters & $\begin{array}{c}\text { Men with } \geq 4 \% \text { morphologically } \\
\text { normal sperm cells }(\mathrm{N}=227)\end{array}$ & $\begin{array}{l}\text { Men with }<4 \% \text { morphologically } \\
\text { normal sperm cells }<4 \%(N=296)\end{array}$ \\
\hline Semen volume $(\mathrm{mL})$ & $227,3.50$ (0.50-11.50), 3.96 \pm 1.78 & $296,3.00(0.75-10.00)^{\star}, 3.45 \pm 1.67$ \\
\hline Total number of spermatozoa in ejaculate $\left(\times 10^{6}\right)$ & $227,100.00(0.40-672.00), 135.48 \pm 107.46$ & $296,42.63(0.25-475.00)^{\star \star}, 62.28 \pm 66.80$ \\
\hline $\begin{array}{l}\text { Total motility (progressive and } \\
\text { non-progressive) (\%) }\end{array}$ & $227,74.00(6.00-92.00), 72.20 \pm 12.93$ & $296,46.00(0.00-87.00)^{\star \star}, 42.87 \pm 19.69$ \\
\hline Immotile spermatozoa (\%) & $227,26.00$ (8.00-94.00), $27.75 \pm 12.93$ & $296,54.00(13.00-100.00)^{\star \star}, 57.13 \pm 19.69$ \\
\hline Eosine-negative spermatozoa: live cells (\%) & $227,83.00$ (12.00-96.00), $81.21 \pm 10.51$ & $296,70.00(0.00-96.00)^{\star \star}, 65.67 \pm 19.06$ \\
\hline TZI & $227,1.45$ (1.17-1.92), $1.46 \pm 0.11$ & $296,1.62(1.20-2.35)^{\star \star}, 1.67 \pm 0.22$ \\
\hline Head defects (\%) & $227,90.00$ (74.00-95.00), 88.93 \pm 3.90 & $296,97.00(83.00-100.00)^{\star \star}, 96.13 \pm 3.33$ \\
\hline Neck and midpiece defects (\%) & $227,29.00$ (15.00-59.00), $29.40 \pm 7.60$ & $296,37.00(9.00-84.00)^{\star \star}, 39.39 \pm 13.16$ \\
\hline Tail defects (\%) & $227,16.00(0.00-52.00), 16.58 \pm 6.81$ & $296,23.00(4.00-74.00)^{\star \star}, 25.63 \pm 12.07$ \\
\hline Spermatozoa with excess residual cytoplasm (\%) & $227,0.00(0.00-12.00), 1.41 \pm 1.98$ & $295,3.00(0.00-60.00)^{\star \star}, 4.21 \pm 5.81$ \\
\hline SDF (\%) & $227,13.00$ (2.00-63.00), $15.50 \pm 8.65$ & $296,22.00(5.00-89.00)^{\star \star}, 25.97 \pm 14.53$ \\
\hline
\end{tabular}

Data are expressed as number of subjects, median (range) and mean \pm SD. *, significant difference with group of men with normal sperm morphology at $\mathrm{P}=0.0002$; ${ }^{*}$, highly significant difference with group of men with normal sperm morphology at $\mathrm{P}<0.00001$ (Mann-Whitney U-test). N, number of subjects; SD, standard deviation; SDF, sperm DNA fragmentation; TZI, teratozoospermia index.

Table 3 Prevalence levels of SDF in study groups

\begin{tabular}{lcc}
\hline Level of SDF $(\%)$ & Men with $\geq 4 \%$ morphologically normal sperm cells, N (\%) & Men with $<4 \%$ morphologically normal sperm cells, N (\%) \\
\hline $0-15$ & $133(58.60)$ & $68(22.97)^{\star \star}$ \\
$16-30$ & $83(36.56)$ & $131(44.26)$ \\
$>30$ & $11(4.84)$ & $97(32.77)^{\star \star}$ \\
\hline
\end{tabular}

${ }^{* *}$, highly significant difference between compared groups at $\mathrm{P}<0.0001$. Chi ${ }^{2}$ test. $\mathrm{N}$, number of subjects; SDF, sperm DNA fragmentation.

Table 4 Odds ratio for SDF levels in study groups

\begin{tabular}{lccc}
\hline Level of SDF (\%) & $\begin{array}{c}\text { Men with } \geq 4 \% \text { morphologically } \\
\text { normal sperm cells, N (\%) }\end{array}$ & $\begin{array}{c}\text { Men with }<4 \% \text { morphologically normal } \\
\text { sperm cells, N (\%) }\end{array}$ & OR (95\% Cl) \\
\hline $0-15$ & $133(58.60)$ & $68(22.97)$ & $0.2175(0.1493-0.3170)^{\star *}$ \\
$16-30$ & $83(36.56)$ & $131(44.26)$ & $1.3774(0.9664-1.9632)$ \\
$>30$ & $11(4.84)$ & $97(32.77)$ & $9.5715(4.9836-18.3829)^{\star *}$ \\
\hline
\end{tabular}

**, highly significant difference between compared groups at $\mathrm{P}<0.0001$. $\mathrm{Chi}^{2}$ test. $95 \% \mathrm{Cl}, 95 \%$ confidential interval; $\mathrm{N}$, number of subjects; OR, odds ratio; SDF, sperm DNA fragmentation. 
tail defects $(\mathrm{P}=0.00001)$; and the percentage of sperm with residual cytoplasm $(\mathrm{P}=0.00022)$ (rank Spearman correlation test) (Table 7).

\section{Discussion}

\section{Detailed sperm structural defects coexist with abnormal nuclear sperm DNA dispersion}

In our study, we were trying to evaluate an association between sperm morphological defects and sperm nuclear DNA fragmentation by comparing the findings from men

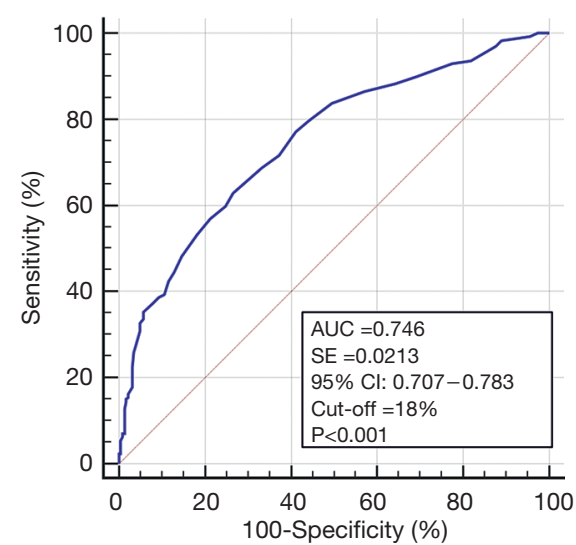

Figure 2 Receiver operating characteristic (ROC) curve. Negative predictive value of sperm DNA fragmentation (SDF) for sperm morphology. $\mathrm{P} \leq 0.05$ : statistical significance between obtained AUC $v s$. $\mathrm{AUC}=0.5$. AUC, area under the curve; SE, standard error; $95 \%$ CI, $95 \%$ confidence interval. with teratozoospermia to those from men with normal sperm morphology. The obtained results suggested that detailed sperm structural defects coexist with abnormal nuclear sperm DNA dispersion. Our data revealed that there were significant differences between men not only in the standard semen analysis but also in the detailed sperm morphological analysis and SDF rate as measured by the Halosperm test. Men with teratozoospermia had a higher percentage of detailed sperm morphological defects and a higher proportion of sperm cells with abnormal chromatin integrity.

It should be highlighted that some authors consider $>30 \%$ of sperm cells with DNA damage as the cut-off for a high risk of infertility $(27,29,31,36-39)$. This is why we used this value as our starting value. In our present group of men with abnormal sperm morphology, $32.77 \%$ of the subjects exhibited a high level of SDF (>30\%), in contrast to $4.84 \%$ of the subjects in the group of men with normal sperm morphology. An inverse relationship was found for the low level of SDF (0-15\%): only $22.97 \%$ of men with teratozoospermia and even $58.60 \%$ of men without teratozoospermia had low levels. These results correspond with the OR showing that men with abnormal sperm morphology had an almost ten-fold higher risk for having an SDF level $>30 \%$ than men with normal sperm morphology. Our results were partially in agreement with data obtained by other authors $(9,10,15,16,37,38)$. Ouimaima et al. (15) showed in their study that there was a clear relationship between sperm morphology and sperm DNA integrity. The authors concluded that good morphological examination is important during routine semen evaluations, which

Table 5 Prevalence of SDF levels in study groups

\begin{tabular}{lcc}
\hline Level of SDF (\%) & Men with $\geq 4 \%$ morphologically normal sperm cells, $\mathrm{N}(\%)$ & Men with $<4 \%$ morphologically normal sperm cells, $\mathrm{N}(\%)$ \\
\hline $0-18$ & $166(73.12)$ & $110(37.16)^{\star \star}$ \\
$>18$ & $61(26.88)$ & $186(62.84)^{\star \star}$
\end{tabular}

${ }^{* *}$, highly significant difference between compared groups at $\mathrm{P}<0.0001$. Chi ${ }^{2}$ test. $\mathrm{N}$, number of subjects; SDF, sperm DNA fragmentation.

Table 6 Odds ratio for SDF levels in study groups

\begin{tabular}{lccc}
\hline Level of SDF (\%) & $\begin{array}{c}\text { Men with } \geq 4 \% \text { morphologically normal } \\
\text { sperm cells, N (\%) }\end{array}$ & $\begin{array}{c}\text { Men with }<4 \% \text { morphologically normal } \\
\text { sperm cells, N (\%) }\end{array}$ & OR (95\%Cl) \\
\hline $0-18$ & $166(73.12)$ & $110(37.16)$ & $0.2173(0.1491-0.3167)^{\star \star}$ \\
$>18$ & $61(26.88)$ & $186(62.84)$ & $4.6015(3.1580-6.7047)^{\star \star}$ \\
\hline
\end{tabular}

**, highly significant difference between compared groups at $\mathrm{P}<0.0001$. $\mathrm{Chi}^{2}$ test. $95 \% \mathrm{Cl}, 95 \%$ confidential interval; $\mathrm{N}$, number of subjects; OR, odds ratio; SDF, sperm DNA fragmentation. 
Table 7 Correlation between SDF and semen characteristics

\begin{tabular}{|c|c|c|}
\hline Parameters & \multicolumn{2}{|c|}{ Total $(\mathrm{N}=523)$} \\
\hline Semen volume $(\mathrm{mL})$ & 0.018 & NS \\
\hline Sperm concentration $\left(\times 10^{6} / \mathrm{mL}\right)$ & -0.315 & 0.00001 \\
\hline Total number of spermatozoa in ejaculate $\left(\times 10^{6}\right)$ & -0.281 & 0.00001 \\
\hline TZI & 0.352 & 0.00001 \\
\hline Head defects (\%) & 0.375 & 0.00001 \\
\hline Neck and midpiece defects (\%) & 0.236 & 0.00001 \\
\hline Tail defects (\%) & 0.298 & 0.00001 \\
\hline Non-progressive motility (\%) & -0.088 & 0.04317 \\
\hline Total motility (progressive and non-progressive) (\%) & -0.522 & 0.00001 \\
\hline Immotile spermatozoa (\%) & 0.522 & 0.00001 \\
\hline Eosine-negative spermatozoa: live cells (\%) & -0.530 & 0.00001 \\
\hline Hos-test-positive spermatozoa: live cells (\%) & $\mathrm{N}=478,-0.527$ & 0.00001 \\
\hline
\end{tabular}

The interpretation of $r_{s}$ value: $<0.2$, lack of linear dependence; $0.2-0.4$, weak dependence; $>0.4-0.7$, moderate dependence; $>0.7-0.9$, strong dependence; $>0.9$, very strong dependence. Statistical significance in rank Spearman correlation was reached when $\mathrm{P}<0.05$. $\mathrm{N}$, number of subjects; SDF, sperm DNA fragmentation; TZI, teratozoospermia index; NS, not significant.

can, in combination with DNA integrity markers, be a perfect predictor of poor semen quality in the laboratory assessment of infertile men. In turn, another authors $(14,37,38)$ found in their study that SDF in subjects with teratozoospermia was significantly higher than in control subjects, which suggested a relationship between abnormal sperm morphology and SDF.

Moreover, our study exhibited significant negative correlations between SDF and the sperm concentration, total sperm count, sperm morphology, progressive and total sperm motility and sperm vitality but a positive correlation with the TZI; percentage of immotile sperm cells; percentages of head, midpiece and tail defects; and the percentage of sperm with residual cytoplasm. Other authors $(15,37,38)$ also indicate similar correlations, but it must be noted that searching for correlations between detailed sperm morphology and sperm chromatin maturity is not often the aim of seminological studies. Only Ouimaima et al. (15) found significant associations between the percentage of atypical forms of spermatozoa and SDF $(\mathrm{r}=-0.733 ; \mathrm{P}<0.001)$ and between head abnormalities and fragmented DNA $(r=0.448 ; \mathrm{P}<0.001)$.

\section{Men with teratozoospermia may have a bigher risk for sperm DNA damage}

A receiver operating characteristic (ROC) curve was generated to determine a potential association between sperm nuclear DNA fragmentation and sperm morphology. The suggested optimal SDF value of $18 \%$ (significant satisfactory negative predictive value) measured by the DNA sperm dispersion test was the best criterion for predicting normal and abnormal sperm morphology. The obtained data revealed that $62.84 \%$ of subjects with teratozoospermia had SDF levels that were $>18 \%$ SDF and that they had up to a five-fold higher risk for $>18 \%$ SDF than men without teratozoospermia. It should be noted that, to the best of our knowledge, this is the first study to determine the predictive value of SDF for sperm morphology using ROC curve analysis. However, many researchers $(9,11-13,15,16,37)$ 
agree that these two parameters are correlated and important in the field of infertility diagnostics. Oumaima et al. (15) concluded that DNA fragmentation is the main pathway leading to morphological defects in sperm. In fact, impaired chromatin compaction that can induce DNA breaks, which can disrupt the DNA backbone, indirectly appears to be the major cause of poor semen morphology, which is partially confirmed and demonstrated by our findings (Figure 1). The results demonstrated by Daris et al. (9) showed that a high percentage of spermatozoa with head abnormalities in sperm samples indicates an elevated degree of DNA fragmentation. They also demonstrated that there was a significant difference in the percentage of sperm cells with normal morphology between sperm samples with low DNA fragmentation and sperm samples with high DNA fragmentation, when a cut-off value of $20 \%$ for DNA fragmentation was used by the authors. The percentages of macrocephalic heads, microcephalic heads, elongated heads, nuclear defects, amorphous heads and tail abnormalities were higher in the group with a degree of DNA fragmentation $\geq 20 \%$. When all head abnormalities were taken together, a statistically significant difference was also established. Agarwal et al. (39) suggested that disturbances in spermatozoa production and maturation may have a dramatic impact on the structural characteristics of semen. They emphasize that sperm morphology is probably the most relevant parameter for traditional semen evaluations, providing information on the fertilization potential. In combination with modern markers of semen quality, sperm morphology may provide the best indication value for poor semen quality in the laboratory assessment of infertile men, which is in line with our suggestion that men with teratozoospermia have a higher risk for sperm DNA damage (Figure 1).

\section{Study limitations}

The present study has some limitations. Firstly, our study group consisted of men attending to the laboratory, which is a research and scientific unit, which means, that the access to research was open and unlimited, any adult man could take part in the research, also volunteers. This is the reason, why our study group contains of only 523 subjects and why have we divided our study group into two groups with regard to sperm morphology, not fertility status, we were not able to get reliable data. Therefore, in our study we did not identify men with teratozoospermia as infertile or fertilethis analysis was done independent of the fertility status of the person. However, teratozoospermia can contribute to infertility, especially when coexists with abnormal sperm chromatin maturity. Secondly, we calculated our threshold for SDF at $18 \%$ as distinguishing between men with teratozoospermia and without teratozoospermia, not $30 \%$, as previously suggested by the manufacturer of the Halosperm $^{\circledR}$ test. It is possible that if we limited the group of men to those with proven fertility and to those with isolated male fertility problems, the threshold value of SDF based on ROC analysis would be different.

\section{Conclusions}

Our findings revealed that the cut-off value for the SDF level in men with teratozoospermia is $18 \%$. This threshold value for SDF differs from that suggested by manufacturerprovided tests, which use an SDF cut-off value of $30 \%$. However, there is no doubt that the suggested optimal value of $18 \%$ measured by the Halosperm test is a criterion to predict normal and abnormal sperm morphology, indicates medical intervention and is useful for implementing the correct therapeutic scenario for men with teratozoospermia. The obtained findings demonstrate that: (I) detailed sperm structural defects coexist with abnormal nuclear sperm DNA dispersion; (II) men with teratozoospermia may have a higher risk for sperm DNA damage; (III) the calculated optimal SDF value of $18 \%$ measured by the DNA sperm dispersion test is the best criterion to predict normal and abnormal sperm morphology.

\section{Acknowledgments}

The authors would like to thank our colleagues of the center for reproductive medicine, their help is most grateful. We also like to thank the patients who participated in this study. Funding: This work was supported by the Pomeranian Medical University in Szczecin, Szczecin, Poland. Grant No. WNoZ-322-04/S/16/2019. We would like to thank all those who helped conducting this study.

\section{Footnote}

Conflicts of Interest: All authors have completed the ICMJE uniform disclosure form (available at http://dx.doi. org/10.21037/tau.2020.01.31). The authors have no conflicts of interest to declare.

Ethical Statement: The authors are accountable for all 
aspects of the work in ensuring that questions related to the accuracy or integrity of any part of the work are appropriately investigated and resolved. The ethics committee of the Pomeranian Medical University, Szczecin, Poland approved the study protocol (ethical authorization number: KB-0012/138/16). All participants provided informed consent.

Open Access Statement: This is an Open Access article distributed in accordance with the Creative Commons Attribution-NonCommercial-NoDerivs 4.0 International License (CC BY-NC-ND 4.0), which permits the noncommercial replication and distribution of the article with the strict proviso that no changes or edits are made and the original work is properly cited (including links to both the formal publication through the relevant DOI and the license). See: https://creativecommons.org/licenses/by-ncnd/4.0/.

\section{References}

1. Agarwal A, Mulgund A, Hamada A, et al. A unique view on male infertility around the globe. Reprod Biol Endocrinol 2015;26;13:37.

2. Cho CL, Agarwal A. Role of sperm DNA fragmentation in male factor infertility: A systematic review. Arab J Urol 2017;16:21-34.

3. Khatun A, Rahman MS, Pan MG. Clinical assessment of the male fertility. Obstet Gynecol Sci 2018;61:179-91.

4. World Health Organization laboratory manual for the Examination and processing of human semen, 5th edition, WHO Press, World Health Organization, Geneva, Switzerland 2010.

5. Agarwal A, Cho CL, Esteves SC. Should we evaluate and treat sperm DNA fragmentation? Curr Opin Obstet Gynecol 2016;28:164-71.

6. Agarwal A, Cho CL, Majzoub A, et al. The Society for Translational Medicine: clinical practice guidelines for sperm DNA fragmentation testing in male infertility. Transl Androl Urol 2017;6:S720-33.

7. Bach PV, Schlegel PN. Sperm DNA damage and its role in IVF and ICSI. Basic Clin Androl 2016;26:15.

8. Küçük N. Sperm DNA and detection of DNA fragmentations in sperm. Turk J Urol 2018;44:1-5.

9. Daris B, Goropevsek A, Hojnik N, et al. Sperm morphological abnormalities as inditators of DNA fragmentation and fertilization in ISCI. Arch Gynecol Obstet 2010;281:363-7.
10. Dehghanpour F, Tabibnejad N, Fesahat F, et al. Evaluation of sperm protamine deficiency and apoptosis in infertile men with idiopathic teratozoospermia. Clin Exp Reprod Med 2017;44:73-8.

11. Franken DR. How accurate is sperm morphology as an indicator of sperm function? Andrologia 2015;47:720-3.

12. Mangiarini A, Paffoni A, Restelli L, et al. Specific sperm defects are differentially correlated with DNA fragmentation in both normozoospermic and teratozoospermic subjects. Andrology 2013;1:838-44.

13. Skowronek F, Casanova G, Alciaturi J, et al. DNA sperm damage correlates with nuclear ultrastructural sperm defects in teratozoospermic men. Andrologia 2012;44:59-65.

14. Tang SS, Gao H, Zhao Y, et al. Aneuploidy and DNA fragmentation in morphologically abnormal sperm. Int J Androl 2010;33:e163-79.

15. Oumaima A, Tesnim A, Zohra H, et al. Investigation on the origin of sperm morphological defects: oxidative attacks, chromatin immaturity, and DNA fragmentation. Environ Sci Pollut Res Int 2018;25:13775-86.

16. Sunanda P, Panda B, Dash C, et al. An illustration of human sperm morphology and their functional ability among different group of subfertile males. Andrology 2018;6:680-9.

17. Holstein AF, Schulze W, Davidoff M. Understanding spermatogenesis is a prerequisite for treatment. Reprod Biol Endocrinol 2003;1:107.

18. Gold HB, Jung YH, Corces VG. Not just heads and tails: The complexity of sperm epigenome. J Biol Chem 2018;293:13815-20.

19. Sakkas D, Seli E, Bizzaro D, et al. Abnormal spermatozoa in the ejaculate: abortive apoptosis and faulty nuclear remodelling during spermatogenesis. Reprod Biomed Online 2003;7:428-32.

20. Sakkas D, Seli E, Manicardi GC, et al. The presence of abnormal spermatozoa in the ejaculate: did apoptosis fail? Hum Fertil (Camb) 2004;7:99-103.

21. Aitken RJ, De Iuliis GN. On the possible origins of DNA damage in human spermatozoa. Mol Hum Reprod 2010;16:3-13.

22. Evgeni E, Charalabopoulos K, Asimakopoulos B. Human sperm DNA fragmentation and its correlation with conventional semen parameters. J Reprod Infertil 2014;15:2-14.

23. Grunewald S, Fitzl G, Springsguth C. Induction of ultra-morphological features of apoptosis in mature and immature sperm. Asian J Androl 2017;19:533-7. 
24. Piasecka M, Gill K, Gączarzewicz D, et al. The significance of the sperm morphology assessment in the seminological diagnostics. In: Piasecka M. editor. Male reproductive system: clinical and experimental studies. Pomeranian Medical University Publishing, 2013:97-123.

25. Wdowiak A, Wdowiak A, Bakalczuk S, et al. Relationship between alcohol consumption and a sperm nuclear DNA fragmentation and pregnancy. Postep Androl Online 2016;3:14-21.

26. Gill K, Jakubik J, Kups M, et al. The impact of sedentary work on sperm nuclear DNA integrity. Folia Histochem Cytobiol 2019;57:15-22.

27. Evenson DP, Larson KL, Jost LK. Sperm chromatin structure assay: its clinical use for detecting sperm DNA fragmentation in male infertility and comparisions with other techniques. J Androl 2002;23:25-43.

28. Bungum, M, Humaidan, P, Axmon, A, et al. Sperm DNA integrity assessment in prediction of assisted reproduction technology outcome. Hum Reprod 2007,22:174-9.

29. Bungum M. Sperm DNA integrity assessment: a new tool in diagnosis and treatment of fertility. Obstet Gynecol Int 2012;2012:531042.

30. Leach M, Aitken RJ, Sacks G. Sperm DNA fragmentation abnormalities in men from couples with a history of recurrent miscarriage. Aust N Z J Obstet Gynaecol 2015;55:379-83.

31. Simon L, Emery BR, Carrell DT. Review: Diagnosis and impact of sperm DNA alterations in assisted reproduction. Best Pract Res Clin Obstet Gynaecol 2017;44:38-56.

32. Abdelbaki SA, Sabry JH, Al-Ad AM, et al. The impact

Cite this article as: Jakubik-Uljasz J, Gill K, Rosiak-Gill A, Piasecka M. Relationship between sperm morphology and sperm DNA dispersion. Transl Androl Urol 2020;9(2):405-415. doi: $10.21037 /$ tau.2020.01.31 of coexisting sperm DNA fragmentation and seminal oxidative stress on the outcome of varicocelectomy in infertile patients: A prospective controlled study. Arab J Urol 2017;15:131-9.

33. Al Omrani B, Al Eisa N, Javed M, et al. Associations of sperm DNA fragmentation with lifestyle factors and semen parameters of Saudi men and its impact on ICSI outcome. Reprod Biol Endocrinol 2018;19;16:49.

34. Vinnakota C, Cree L, Peek J et al. Incidence of high sperm DNA fragmentation in a targeted population of subfertile men. Syst Biol Reprod Med 2019;65:451.

35. Yang H, Li G, Jin H, et al. The effect of sperm DNA fragmentation index on assisted reproductive technology outcomes and its relationship with semen parameters and lifestyle. Transl Androl Urol 2019;8:356-65.

36. Bounartzi T, Dafopoulos K, Anifandis G, et al. Pregnancy prediction by free sperm DNA and sperm DNA fragmentation in semen specimens of IVF/ICSI-ET patients. Hum Fertil (Camb) 2016;19:56-62.

37. Cissen M, Wely MV, Scholten I, et al. Measuring sperm DNA fragmentation and clinical outcomes of medically assisted reproduction: a systematic review and metaanalysis. PLoS One 2016;10;11:e0165125.

38. Evenson DP. Evaluation of sperm chromatin structure and DNA strand breaks is an important part of clinical male fertility assessment. Transl Androl Urol 2017;6:S495-500.

39. Agarwal A, Tvrda E, Sharma E. Relationship amongst teratozoospermia, seminal oxidative stress and male infertility. Reprod Biol Endocrinol 2014;27;12:45. 